\title{
The Hermitian Kirchhoff Index and Robustness of Mixed Graph
}

\author{
Wei Lin $\mathbb{D}^{1},{ }^{1}$ Shuming Zhou $\mathbb{D}^{2,3}$ Min Li ${ }^{2},^{2,3}$ Gaolin Chen $\mathbb{D}^{2,3}$ and Qianru Zhou $\mathbb{D}^{2,3}$ \\ ${ }^{1}$ Concord University College, Fujian Normal University, Fuzhou, Fujian 350117, China \\ ${ }^{2}$ College of Mathematics and Statistics, Fujian Normal University, Fuzhou, Fujian 350117, China \\ ${ }^{3}$ Center for Applied Mathematics of Fujian Province, Fujian Normal University, Fuzhou, Fujian 350117, China
}

Correspondence should be addressed to Shuming Zhou; zhoushuming@fjnu.edu.cn

Received 24 February 2021; Revised 7 May 2021; Accepted 5 June 2021; Published 30 June 2021

Academic Editor: Mijia Yang

Copyright (C) 2021 Wei Lin et al. This is an open access article distributed under the Creative Commons Attribution License, which permits unrestricted use, distribution, and reproduction in any medium, provided the original work is properly cited.

\begin{abstract}
Large-scale social graph data poses significant challenges for social analytic tools to monitor and analyze social networks. The information-theoretic distance measure, namely, resistance distance, is a vital parameter for ranking influential nodes or community detection. The superiority of resistance distance and Kirchhoff index is that it can reflect the global properties of the graph fairly, and they are widely used in assessment of graph connectivity and robustness. There are various measures of network criticality which have been investigated for underlying networks, while little is known about the corresponding metrics for mixed networks. In this paper, we propose the positive walk algorithm to construct the Hermitian matrix for the mixed graph and then introduce the Hermitian resistance matrix and the Hermitian Kirchhoff index which are based on the eigenvalues and eigenvectors of the Hermitian Laplacian matrix. Meanwhile, we also propose a modified algorithm, the directed traversal algorithm, to select the edges whose removal will maximize the Hermitian Kirchhoff index in the general mixed graph. Finally, we compare the results with the algebraic connectivity to verify the superiority of the proposed strategy.
\end{abstract}

\section{Introduction}

Network science has been an emerging area in big data, cyberspace security, and artificial intelligence [1-4]. Many real-world networks are mixed, such as citation networks, web page link networks, e-mail networks, and information diffusion networks. Mixed networks, just like the underlying one, emerge in the fields of multiagent systems $[5,6]$, biology [7], and society [8]. Many efficient algorithms have been proposed to resolve the function and topological structure of mixed networks, such as decentralized optimization [9], node anomaly detection algorithm [10], embedding algorithm [11], and magnetic Laplacian [12]. Undoubtedly, further analysis of mixed networks has become an indispensable topic in the research of network science and interdisciplinary.

1993, Klein and Randić first proposed resistance distance [13]. Xiao and Gutman [14] uncovered some properties of a resistance matrix. Combining the normalized Laplacian matrix and the transition probability matrix, Chen and Zhang [15] proposed a new degree-Kirchhoff index. Atik et al. [16] extended the resistance distance to the weighted graph. Up to this day, resistance distance has many important applied areas, such as phase transition [17], silicate network [18], electrical network [19, 20], and complex networks [21]. Compared to the calculation of eigenvalues and eigenvectors, the advantage of resistance distance as a metric is that it better reflects the overall nature of the network, and it can reduce the computational complexity in a large-scale network. To the best of my knowledge, the research of resistance distance is mainly carried out in the unweighted and undirected networks. There are few research studies about the resistance distance in mixed networks, which is the motivation of this paper. The focus in this paper is to establish a new resistance distance metric for the mixed networks.

As it is known to all, the study of the resistance distance is inseparable from matrix theory. Matrix theory, including Hermitian adjacency matrix [22, 23], Laplacian matrix $[24,25]$, nonnegative matrix factorization [26], and conditional probability matrix [27], is a powerful tool to deal with graph topology structure and function because it can 
transform complex calculations into a series of four operations, which are easy to improve computational performance. On the basis of [23], Mohar [28] proposed the Hermitian matrix of the second kind. Yu et al. [29-31] have been devoted to the study of Hermitian spectral theory for mixed graphs. The extension from the underlying graph to the mixed graph is actually an extension from the real field to the complex field. The importance of the Hermitian matrix is becoming increasingly prominent. The matrix construction depends on the graph direction, so how to give the graph direction that meets the conditions is also an urgent problem to be solved. With the development of the Hermitian matrix, more and more models and strategies have been put forward [32-34]. The Randic index has been widely used in network centrality, clustering algorithm, network connectivity, and robustness [35-37]. Meo et al. [38] estimated graph robustness through the Randic Index. Along the direction of the Hermitian matrix, we attempt to introduce the Hermitian resistance matrix and the Kirchhoff index of the general mixed graph to estimate robustness of the mixed graph.

In this paper, analogue to pseudoinverse matrix of the Laplacian matrix for underlying graph, we extend the corresponding invertible matrix $X_{\mathscr{H}}$ for mixed graph. Our main contributions are as follows:

(1) We propose the Hermitian resistance matrix and the Hermitian Kirchhoff index for positive mixed graph and then establish a formula for the determinant and the inverse of the Hermitian resistance matrix

(2) We propose a positive walk algorithm for the general mixed graph

(3) Robustness of the general mixed graph is evaluated by the modified directed traversal algorithm based on the Hermitian Kirchhoff index

The remainder of the paper is organized as follows. Section 1 presents related work of the resistance matrix and the Kirchhoff index. Section 2 introduces the Hermitian Laplacian matrix. In Section 3, we define the Hermitian resistance matrix $R_{\mathscr{H}}$ and compute its determinant in the positive mixed graph. In Section 4, we derive formulae for inverse of the Hermitian resistance matrix. Section 5 characterizes the Hermitian Kirchhoff index in the positive mixed graph. The statistical experimental analysis in the general mixed graph is shown in Section 6. Section 7 concludes the paper.

\section{Hermitian Laplacian Matrix}

Let $G$ be a mixed graph with a finite vertices set $V(G)$ and a subset $E(G) \subseteq V(G) \times V(G)$. The edge set $E(G)$ is the union of undirected edges and the directed edges. For convenience, we define the undirected edge as $v_{i} \leftrightarrow v_{j}$ and the directed edge is $v_{i} \longrightarrow v_{j}$ if the orientation is from $v_{i}$ to $v_{j}$. The diagonal matrix $D=\operatorname{diag}\left(d_{1}, d_{2}, \ldots, d_{n}\right)$ is the degree matrix, where $d_{i}$ is the degree of $v_{i}$ of the underlying graph $\Omega(G)$. We denote $H^{*}=(\bar{H})^{T}$. Then, $H$ is a Hermitian matrix if $H^{*}=H$. The Hermitian adjacency matrix of a mixed graph is the matrix $\mathscr{H} \in \mathbb{C}^{V \times V}[22,23]$, whose element

$$
\eta_{i j}=\left\{\begin{array}{lll}
1, & \text { if } & v_{i} \leftrightarrow v_{j}, \\
i, & \text { if } & v_{i} \longrightarrow v_{j}, \\
-i, & \text { if } & v_{i} \leftarrow v_{j}, \\
0, & \text { otherwise. }
\end{array}\right.
$$

The value of a mixed walk $T=v_{1} v_{2}, \ldots, v_{k}$ in $G$ is $\eta(T)=\eta_{12} \eta_{23}, \ldots, \eta_{(k-1) k}$. A mixed walk is positive (negative) if $\eta(T)=1(\eta(T)=-1)$.

Lemma 1 (see [30]). A mixed graph $G$ is positive if and only if, for any two vertices $v_{1}$ and $v_{2}$, all mixed paths from $v_{1}$ to $v_{2}$ have the same value.

Lemma 2 (see [31]). Let $G$ be a connected mixed graph on vertices $v_{1} v_{2}, \ldots, v_{n}$. If $L_{\mathscr{H}}=D-\mathscr{H}$ is a positive semidefinite matrix and singular, then 0 is a simple eigenvalue with an eigenvector:

$$
1_{\mathscr{H}}=\left(1, \overline{\eta\left(T_{2}\right)}, \overline{\eta\left(T_{3}\right)}, \ldots, \overline{\eta\left(T_{n}\right)}\right)^{T},
$$

where $T_{i}$ is a 1 -i-walk in $G$.

The Hermitian Laplacian eigenvalues and orthogonal eigenvectors will be denoted by $\lambda_{i}$ and $u_{i}=\left(u_{i 1}, u_{i 2}, \ldots, u_{i n}\right)^{T}$, respectively. Denote the unitary matrix:

$$
U_{\mathscr{H}}=\left(u_{1}, u_{2}, u_{3}, \ldots, u_{n}\right),
$$

where $u_{n}=1 / \sqrt{n} 1_{\mathscr{H}}$.

Then, we have

$$
U_{\mathscr{H}}^{*} L_{\mathscr{H}} U_{\mathscr{H}}=\operatorname{diag}\left[\lambda_{1}, \ldots, \lambda_{n-1}, 0\right],
$$

and

$$
U_{\mathscr{H}} U_{\mathscr{H}}^{*}=U_{\mathscr{H}}^{*} U_{\mathscr{H}}=I
$$

i.e.,

$$
\sum_{k=1}^{n} u_{k i} \overline{u_{k j}}=\sum_{k=1}^{n} \overline{u_{i k}} u_{j k}=\left\{\begin{array}{lll}
1, & \text { if } & i=j \\
0, & \text { if } & i \neq j .
\end{array}\right.
$$

\section{Hermitian Resistance Matrix in Positive Mixed Graph}

In this section, we establish the relationship between the Hermitian resistance matrix and the Hermitian Laplacian matrix in the positive mixed graph.

Lemma 3 (see [22]). Let $G$ be a mixed graph. Then, the following are equivalent:

(1) $G$ is a positive

(2) $G \sim G_{u}$

Lemma 4 (see [30]). Let $G$ be a positive mixed graph. Then, the following statements hold: 
(1) $S P_{\mathscr{H}}(G)=S P_{A}\left(G_{u}\right)$, where $S P_{A}\left(G_{u}\right)$ is the spectrum of the adjacency matrix of $G_{u}$

(2) $S P_{L_{y y}}(G)=S P_{L}\left(G_{u}\right)$, where $S P_{L}\left(G_{u}\right)$ is the spectrum of the Laplacian matrix of $G_{u}$

By Lemma 3 and Lemma 4, we give the definition of $n \times n$ orientation matrix $O_{\mathscr{H}} \in \mathbb{C}$ :

$$
O_{\mathscr{H}}=\left(\begin{array}{llll}
1 & & & \\
& \overline{\eta\left(T_{2}\right)} & & \\
& & \ddots & \\
& & & \overline{\eta\left(T_{n}\right)}
\end{array}\right) .
$$

Then, we establish the relationship between the Hermitian matrix and the corresponding matrix in the underlying graph by the orientation matrix $O_{\mathscr{H}}$.

Lemma 5 (see [22]). Let $G$ be a positive mixed graph on $n$ vertices and $\mathscr{H}$ and $A$ be the Hermitian adjacency matrix and the adjacency matrix, respectively. For a diagonal matrix $O_{\mathscr{H}}=\operatorname{diag}\left(1, \overline{\eta\left(T_{2}\right)}, \ldots, \overline{\eta\left(T_{n}\right)}\right)$, we have

$$
\mathrm{O}_{\mathscr{H}}^{*} \mathscr{H} \mathrm{O}_{\mathscr{H}}=A .
$$

Corollary 1. Let $G$ be a positive mixed graph on $n$ vertices, $L_{\mathscr{H}}$ be the Hermitian Laplacian matrix, and $L$ be the Laplacian matrix of the underlying graph. For the orientation matrix $O_{\mathscr{H}}=\operatorname{diag}\left(1, \overline{\eta\left(T_{2}\right)}, \ldots, \overline{\eta\left(T_{n}\right)}\right)$, we have $O_{\mathscr{H}}^{*} L_{\mathscr{H}} O_{\mathscr{H}}=L$.

Now, we denote

$$
J_{\mathscr{H}}=1_{\mathscr{H}} 1_{\mathscr{H}}^{*} .
$$

Therefore,

$$
U_{\mathscr{H}}^{*} J_{\mathscr{H}} U_{\mathscr{H}}=\left(U_{\mathscr{H}}^{*} 1 \mathscr{H}\right)\left(U_{\mathscr{H}}^{*} 1_{\mathscr{H}}\right)^{*}=\operatorname{diag}[0,0, \ldots, n] .
$$

By equations (4) and (10), we have

$$
U_{\mathscr{H}}^{*}\left(L_{\mathscr{H}}+\frac{1}{n} J_{\mathscr{H}}\right) U_{\mathscr{H}}=\operatorname{diag}\left[\lambda_{1}, \ldots, \lambda_{n-1}, 1\right] .
$$

The matrix $L_{\mathscr{H}}+1 / n J_{\mathscr{H}}$ is nonsingular, and we define its inverse

$$
X_{\mathscr{H}}=\left(L_{\mathscr{H}}+\frac{1}{n} J_{\mathscr{H}}\right)^{-1}
$$

Similarly, we have the following corollary.

Corollary 2. Let $G$ be a positive mixed graph on $n$ vertices and $X=(L+1 / n J)^{-1}$, where $J$ denote the square with all entries equal to 1 . Then, $X_{\mathscr{H}}$ is Hermitian and

$$
O_{\mathscr{H}}^{*} X_{\mathscr{H}} O_{\mathscr{H}}=X \text {. }
$$

Now, we apply this process to the resistance matrix.

Theorem 1. Let $G$ is a positive mixed graph and $R$ is the resistance matrix of the underlying graph; then, the Hermitian resistance matrix is

$$
R_{\mathscr{H}}=O_{\mathscr{H}} R O_{\mathscr{H}}^{*} .
$$

Proof 1. By Theorem 3 and the definition of resistance matrix, we have

$$
\begin{aligned}
O_{\mathscr{H}} R O_{\mathscr{H}}^{*}= & \left(\begin{array}{ccc}
x_{11} & & \\
& \ddots & \\
& & x_{n n}
\end{array}\right) O_{\mathscr{H}} J O_{\mathscr{H}}^{*} \\
& +O_{\mathscr{H}} J O_{\mathscr{H}}^{*}\left(\begin{array}{lll}
x_{11} & & \\
& \ddots & \\
& & x_{n n}
\end{array}\right)-O_{\mathscr{H}} X O_{\mathscr{H}}^{*} \\
= & \left(\begin{array}{ccc}
x_{11} & & \\
& \ddots & \\
& & x_{n n}
\end{array}\right) J_{\mathscr{H}}+J_{\mathscr{H}}\left(\begin{array}{lll}
x_{11} & & \\
& \ddots & \\
& & x_{n n}
\end{array}\right)-X_{\mathscr{H}} \\
=R_{\mathscr{H}}, &
\end{aligned}
$$

where $x_{i i}$ is the diagonal member of $X$.

In view of Theorem 1 , we have 
$U_{\mathscr{H}}^{*} R_{\mathscr{H}}^{*} U_{\mathscr{H}}=U_{\mathscr{H}}^{*}\left[\left(\begin{array}{ccc}x_{11} & & \\ & \ddots & \\ & & x_{n n}\end{array}\right) J_{\mathscr{H}}+J_{\mathscr{H}}\left(\begin{array}{ccc}x_{11} & \\ & \ddots & \\ & & \\ & & x_{n n}\end{array}\right)-2 X_{\mathscr{H}}\right] U_{\mathscr{H}}$,

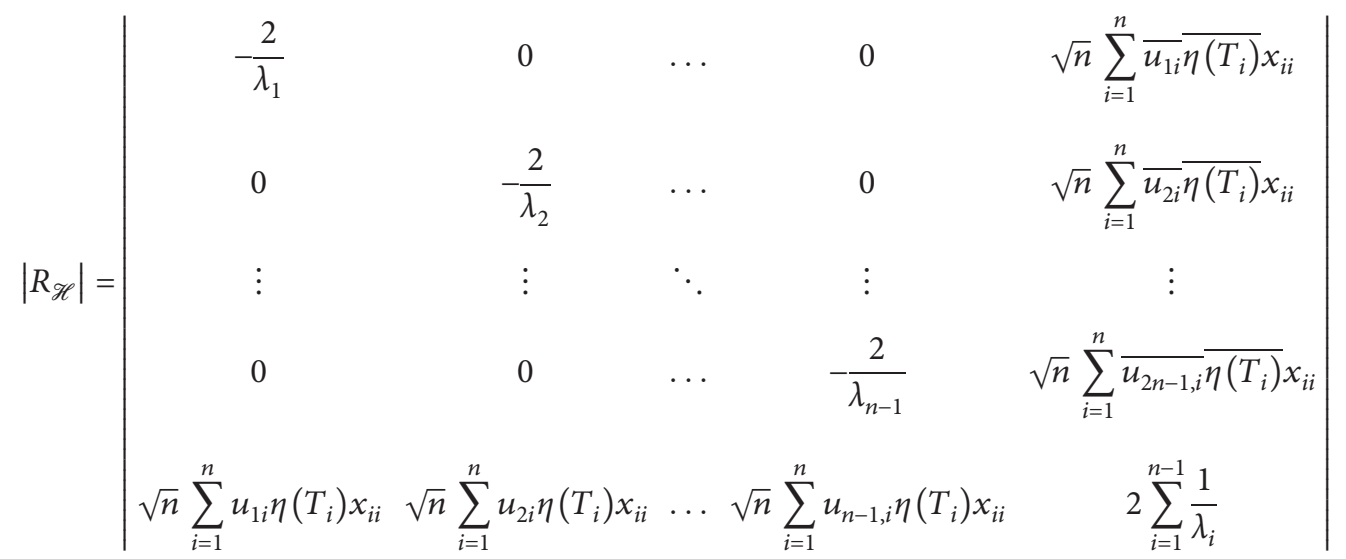

$$
\begin{aligned}
& =\left|\begin{array}{ccccc}
-\frac{2}{\lambda_{1}} & 0 & \ldots & 0 & \sqrt{n} \sum_{i=1}^{n} \overline{u_{1 i}} \overline{\eta\left(T_{i}\right)} x_{i i} \\
0 & -\frac{2}{\lambda_{2}} & \ldots & 0 & \sqrt{n} \sum_{i=1}^{n} \overline{u_{2 i}} \overline{\eta\left(T_{i}\right)} x_{i i} \\
\vdots & \vdots & \ddots & \vdots & \vdots \\
0 & 0 & \ldots & -\frac{2}{\lambda_{n-1}} & \sqrt{n} \sum_{i=1}^{n} \overline{u_{2 n-1, i}} \overline{\eta\left(T_{i}\right)} x_{i i} \\
0 & 0 & \ldots & 0 & S_{\mathscr{H}}+2 \sum_{i=1}^{n-1} \frac{1}{\lambda_{i}}
\end{array}\right|,
\end{aligned}
$$

where

Therefore, we have

$$
\begin{aligned}
S_{\mathscr{H}} & =\frac{n}{2} \sum_{i=1}^{n-1} \lambda_{i}\left(\sum_{k=1}^{n} \overline{u_{i k}} \overline{\eta\left(T_{k}\right)} x_{k k}\right)\left(\sum_{t=1}^{n} u_{i t} \eta\left(T_{t}\right) x_{t t}\right) \\
& =\frac{n}{2} \sum_{k=1}^{n} \sum_{t=1}^{n} \overline{\eta\left(T_{k}\right)} x_{k k} \eta\left(T_{t}\right) x_{t t}\left(\sum_{i=1}^{n-1} u_{i t} \lambda_{i} \overline{u_{i k}}\right) \\
& =\frac{n}{2} \sum_{t=1}^{n} \sum_{k=1}^{n} \eta\left(T_{t}\right) x_{t t} \overline{\eta\left(T_{k}\right)} x_{k k}\left(L_{\mathscr{H}}\right)_{t k} \\
& =\frac{n}{2}\left(x_{i i}, x_{22}, \ldots, x_{n n}\right) D_{\mathscr{H}}^{*} L_{\mathscr{H}} D_{\mathscr{H}}\left(x_{i i}, x_{22}, \ldots, x_{n n}\right) \prime .
\end{aligned}
$$

$$
\left|R_{\mathscr{H}}\right|=(-1)^{n-1} \frac{2^{n-1}}{\prod_{i=1}^{n-1} \lambda_{i}\left(S_{\mathscr{H}}+2 \sum_{i=1}^{n-1} \frac{1}{\lambda_{i}}\right)=(-1)^{n-1} \frac{2^{n-1}}{n t(G)}\left(S_{\mathscr{H}}+2 \sum_{i=1}^{n-1} \frac{1}{\lambda_{i}}\right)}
$$


where $t(G)$ is the number of spanning trees of the $G$.

\section{Inverse of the Hermitian Resistance Matrix}

In this section, we will show that the Hermitian resistance matrices are nonsingular and derive a formula for the inverse of them.

For $i=1,2, \ldots, n$, we define the $n \times 1$ vector $\omega=\left(\omega_{1}, \omega_{2}, \ldots, \omega_{n}\right)^{T}$, such that

$$
\omega_{i}=2 \overline{\eta\left(T_{i}\right)}-\sum_{j \sim i} \overline{\eta\left(T_{j}\right)} r_{\mathscr{H}}(i, j)
$$

Theorem 2. Let $G$ be a positive mixed graph on $n$ vertices and $L_{\mathscr{H}}$ be the Hermitian Laplacian matrix associated with $G$. If the $\tilde{X}_{\mathscr{H}}=\operatorname{diag}\left(x_{11}, x_{22}, \ldots, x_{n n}\right)$ and $\omega$ are defined as above, then

$$
\omega=L_{\mathscr{H}} \tilde{X}_{\mathscr{H}} 1_{\mathscr{H}}+\frac{2}{n} 1_{\mathscr{H}}
$$

Proof 2. Let $d_{i}$ be the degree of vertex $i$. According to $\left(L_{\mathscr{H}}+1 / n J_{\mathscr{H}}\right) X_{\mathscr{H}}^{*}=I$, we have

$$
d_{i} x_{i i}-\eta\left(T_{i}\right) \sum_{j \sim i} \overline{\eta\left(T_{j}\right)} \overline{x_{i j}}+\frac{1}{n} \eta\left(T_{i}\right) \sum_{j=1}^{n} \overline{\eta\left(T_{j}\right)} \overline{x_{i j}}=1 .
$$

Then,

$$
d_{i i} x_{i i}-\eta\left(T_{i}\right) \sum_{j \sim i} \overline{\eta\left(T_{j}\right)} \overline{x_{i j}}+\frac{1}{n}=1 .
$$

Thus,

$$
\sum_{j \sim i} \overline{\eta\left(T_{j}\right)} \overline{x_{i j}}=\overline{\eta\left(T_{i}\right)} d_{i i} x_{i i}+\overline{\eta\left(T_{i}\right)} \frac{1}{n}-\overline{\eta\left(T_{i}\right)} .
$$

Now, for $i=1,2, \ldots, n$, we have

$$
\begin{aligned}
\omega_{i} & =2 \overline{\eta\left(T_{i}\right)}-\sum_{j \sim i} \eta\left(T_{j}\right) r_{\mathscr{H}}(i, j) \\
& =2 \overline{\eta\left(T_{i}\right)}-\sum_{j \sim i} \overline{\eta\left(T_{j}\right)} r_{\mathscr{H}}(i, j) \\
& =2 \overline{\eta\left(T_{i}\right)}-\sum_{j \sim i}\left(\overline{\eta\left(T_{i}\right)} x_{i i}+\overline{\eta\left(T_{i}\right)} x_{j j}-2 \overline{\eta\left(T_{j}\right)} x_{i j}\right) \\
& =2 \overline{\eta\left(T_{i}\right)}-\overline{\eta\left(T_{i}\right)} d_{i} x_{i i}-\overline{\eta\left(T_{i}\right)} \sum_{j \sim i} x_{j j}+2 \sum_{j \sim i} \overline{\eta\left(T_{j}\right)} x_{i j} \\
& =\overline{\eta\left(T_{i}\right)} d_{i} x_{i i}-\overline{\eta\left(T_{i}\right)} \sum_{j \sim i} x_{j j}+\frac{2}{\eta} \overline{\eta\left(T_{i}\right)}[\mathrm{By}(24)] .
\end{aligned}
$$

Hence, $\omega=L_{\mathscr{H}}^{*} \tilde{X}_{\mathscr{H}} 1_{\mathscr{H}}+2 / n 1_{\mathscr{H}}$.

Now, by Theorem 2, we have

$$
1_{\mathscr{H}}^{*} \omega=\frac{2}{n} 1_{\mathscr{H}}^{*} 1_{\mathscr{H}}=2 .
$$

Theorem 3. Let $G$ be a positive mixed graph on $n$ vertices. Let $L_{\mathscr{H}}$ and $R_{\mathscr{H}}$ be the Hermitian Laplacian matrix and the Hermitian resistance matrix, respectively. If the $n \times 1$ vector $\widetilde{x_{\mathscr{H}}}=\widetilde{X_{\mathscr{H}}} 1_{\mathscr{H}}$, then

$$
\omega^{*} R_{\mathscr{H}} \omega=2 \widetilde{x_{\mathscr{H}}}{ }^{*} L_{\mathscr{H}} \widetilde{x_{\mathscr{H}}}+\frac{8}{n} \operatorname{trace}\left(L_{\mathscr{H}}^{\dagger}\right)
$$

Proof 3. By Theorem 2, we have

$$
\begin{aligned}
\omega^{*} R_{\mathscr{H}} \omega= & \left(1_{\mathscr{H}}^{*} \widetilde{X_{\mathscr{H}}} L_{\mathscr{H}}^{*}+\frac{2}{n} 1_{\mathscr{H}}^{*}\right) R_{\mathscr{H}}\left(L_{\mathscr{H}} \widetilde{X_{\mathscr{H}}} 1_{\mathscr{H}}+\frac{2}{n} 1_{\mathscr{H}}\right) \\
= & 1_{\mathscr{H}}^{*} \widetilde{X_{\mathscr{H}}} L_{\mathscr{H}}^{*} R_{\mathscr{H}} L_{\mathscr{H}} \widetilde{X_{\mathscr{H}}} 1_{\mathscr{H}}+\frac{2}{n} 1_{\mathscr{H}}^{*} R_{\mathscr{H}} L_{\mathscr{H}} \widetilde{X_{\mathscr{H}}} 1_{\mathscr{H}} \\
& +\frac{2}{n} 1_{\mathscr{H}}^{*} \widetilde{X_{\mathscr{H}}} L_{\mathscr{H}}^{*} R_{\mathscr{H}} 1_{\mathscr{H}}+\frac{4}{n^{2}} 1_{\mathscr{H}}^{*} R_{\mathscr{H}} 1_{\mathscr{H}} .
\end{aligned}
$$

In view of Theorem 1 , we obtain

$L_{\mathscr{H}}^{*} R_{\mathscr{H}} L_{\mathscr{H}}=-2 L_{\mathscr{H}}^{*} X_{\mathscr{H}} L_{\mathscr{H}}=-2 L_{\mathscr{H}}^{*} L_{\mathscr{H}}^{\dagger} L_{\mathscr{H}}=-2 L_{\mathscr{H}}$.

That is,

$$
1_{\mathscr{H}}^{*} \widetilde{X_{\mathscr{H}}}\left(L_{\mathscr{H}}^{*} R_{\mathscr{H}} L_{\mathscr{H}}\right) \widetilde{X_{\mathscr{H}}} 1_{\mathscr{H}}=-2{\widetilde{x_{\mathscr{H}}}}^{*} L_{\mathscr{H}} \widetilde{x_{\mathscr{H}}} .
$$

Then,

$$
\frac{2}{n} 1_{\mathscr{H}}^{*} \widetilde{X_{\mathscr{H}}} L_{\mathscr{H}}^{*} R_{\mathscr{H}} 1_{\mathscr{H}}=2 \widetilde{x_{\mathscr{H}}}{ }^{*} L_{\mathscr{H}} \widetilde{x_{\mathscr{H}}}
$$

In a similar way,

$$
\frac{2}{n} 1_{\mathscr{H}}^{*} R_{\mathscr{H}} L_{\mathscr{H}} \widetilde{X_{\mathscr{H}}} 1_{\mathscr{H}}=2 \widetilde{x_{\mathscr{H}}}{ }^{*} L_{\mathscr{H}} \widetilde{x_{\mathscr{H}}}
$$

Finally,

$$
\frac{4}{n^{2}} 1_{\mathscr{H}}^{*} R_{\mathscr{H}} 1_{\mathscr{H}}=\frac{8}{n}\left(\operatorname{trace} \widetilde{X_{\mathscr{H}}}-1\right)=\frac{8}{n} \operatorname{trace} L_{\mathscr{H}}^{\dagger} .
$$

Applying equations (32)-(35), we obtain

$$
\omega^{*} R_{\mathscr{H}} \omega=2 \widetilde{x_{\mathscr{H}}}{ }^{*} L_{\mathscr{H}} \widetilde{x_{\mathscr{H}}}+\frac{8}{n} \operatorname{trace}\left(L_{\mathscr{H}}^{\dagger}\right)
$$

Obviously, $\omega^{*} R_{\mathscr{C}} \omega$ is a positive definite matrix.

Next, we show that the Hermitian resistance matrix is nonsingular and establish the inverse of them.

Theorem 4. Let $G$ be a positive mixed graph on $n$ vertices. Let $L_{\mathscr{H}}$ and $R_{\mathscr{H}}$ be the Hermitian Laplacian Matrix and the Hermitian resistance matrix, respectively. Then, $R_{\mathscr{H}}$ is nonsingular and

$$
R_{\mathscr{H}}^{-1}=-\frac{1}{2} L_{\mathscr{H}}+\frac{1}{\omega^{*} R_{\mathscr{H}} \omega} \omega \omega^{*} .
$$


Proof 4. By Theorem 1, we have

$$
\begin{aligned}
L_{\mathscr{H}}^{*} R_{\mathscr{H}}+2 I & =L_{\mathscr{H}}^{*} \tilde{X}_{\mathscr{H}} J_{\mathscr{H}}+\frac{2}{n} J_{\mathscr{H}}=\omega 1_{\mathscr{H}}^{*}, \\
\left(L_{\mathscr{H}}^{*} R_{\mathscr{H}}+2 I\right)^{*} \omega & =\omega 1_{\mathscr{H}}^{*} \omega=2 \omega .
\end{aligned}
$$

Then,

$$
L_{\mathscr{H}}^{*} R_{\mathscr{C}} \omega=0
$$

By Theorem $1, R_{\mathscr{H}} \omega$ is nonzero. In view of $L_{\mathscr{H}}^{*} 1_{\mathscr{H}}=0$, there exists a nonzero scalar $\beta$ such that

$$
\begin{aligned}
R_{\mathscr{H}} \omega & =\beta 1_{\mathscr{H}}, \\
\beta & =\frac{1}{2} \omega^{*} R_{\mathscr{H}} \omega .
\end{aligned}
$$

That is,

$$
R_{\mathscr{H}} \omega=\frac{\omega^{*} R_{\mathscr{H}} \omega}{2} 1_{\mathscr{H}}
$$

Hence,

$$
\begin{aligned}
\left(-\frac{1}{2} L_{\mathscr{H}}^{*}+\frac{\omega \omega^{*}}{\omega^{*} R_{\mathscr{H}} \omega}\right) R_{\mathscr{H}}= & -\frac{1}{2} L_{\mathscr{H}}^{*} R_{\mathscr{H}}+\frac{\omega \omega^{*} R_{\mathscr{H}}}{\omega^{*} R_{\mathscr{H}} \omega} \\
= & -\frac{1}{2} L_{\mathscr{H}}^{*} R_{\mathscr{H}} \\
& +\frac{\omega 1_{\mathscr{H}}^{*}}{\omega^{*} R_{\mathscr{H}} \omega} \frac{\omega^{*} R_{\mathscr{H}} \omega}{2}[\text { By }(39)] \\
= & -\frac{1}{2} L_{\mathscr{H}}^{*} R_{\mathscr{H}}+\frac{1}{2} \omega 1_{\mathscr{H}}^{*} \\
= & I .[\text { By }(36)] .
\end{aligned}
$$

Therefore, the matrix $R_{\mathscr{H}}$ is nonsingular, and $R_{\mathscr{H}}^{-1}=-1 / 2 L_{\mathscr{H}}+1 / \omega^{*} R_{\mathscr{H}} \omega \omega \omega^{*}$.

\section{Hermitian Kirchhoff Index in Positive Mixed Graph}

In this section, we establish the Hermitian Kirchhoff index in terms of the spectrum of $L_{\mathscr{H}}$ in a positive mixed graph.

Theorem 5. Let $G$ be a positive mixed graph on $n$ vertices. Then, the Hermitian Kirchhoff index is

$$
K f=\sum_{i<j} \eta\left(T_{i}\right) \overline{\eta\left(T_{j}\right)} r_{\mathscr{H}}(i, j)=n \sum_{k=1}^{n-1} \frac{1}{\lambda_{k}} .
$$

Proof 5. By Theorem 1, we have

$$
\begin{aligned}
K f & =\sum_{i<j} \eta\left(T_{i}\right) \overline{\eta\left(T_{j}\right)} r_{\mathscr{H}}(i, j) \\
& =\frac{1}{2} \sum_{i=1}^{n} \sum_{j=1}^{n} r(i, j) \\
& =n \sum_{k=1}^{n-1} \frac{1}{\lambda_{k}} .
\end{aligned}
$$

\section{Experimental Analysis of Hermitian Kirchhoff Index in General Mixed Graph}

According to Lemma 5, we propose a positive walk algorithm (see Algorithm 1) to give a positive direction for a given undirected graph $G$.

In the real world, not all mixed graphs are positive. If $L_{\mathscr{H}}$ is nonsingular, we compute the Hermitian resistance matrix by the Moore-Penrose generalized inverse $L^{\dagger}$ of the Hermitian Laplacian matrix of the mixed graph:

$$
r_{\mathscr{H}}(i, j)=\left(L_{\mathscr{H}}^{\dagger}\right)_{i i}+\left(L_{\mathscr{H}}^{\dagger}\right)_{j j}-\left(L_{\mathscr{H}}^{\dagger}\right)_{i j}-\left(L_{\mathscr{H}}^{\dagger}\right)_{j i}
$$

We establish the Hermitian Kirchhoff index in terms of the Hermitian resistance matrix in a mixed graph.

Theorem 6. Let $G$ be a mixed graph on $n$ vertices. Then, the Hermitian Kirchhoff index is

$$
K f=\sum_{i<j} r_{\mathscr{H}}(i, j) .
$$

There is a close relationship between the Kirchhoff index and the robustness of network, for example, the bigger the Kirchhoff index, the lower the ability of network to function in an emergency situation. The Kirchhoff index and the robustness of the network are negative correlation. Further more, algebraic connectivity is also widely used to measure the robustness of networks; however, algebraic connectivity is positively correlated with robustness. That is to say, algebraic connectivity is negatively correlated with the Kirchhoff index. Next, we will use Hermitian algebraic connectivity and Hermitian Kirchhoff index to measure the robustness of the general mixed graph, as a comparison of different robustness measures. In order to verify the rationality and applicability of our suggestion, we propose a directed traversal algorithm (see Algorithm 2) to obtain the edge set $E_{\max }$ whose removal from a mixed graph $G$ will maximize the Hermitian Kirchhoff index. Then, we compare it with the results of algebraic connectivity. Peng et al. [39] investigated the robustness of undirected mesh graphs. We generalize mixed mesh graphs to random graphs.

We take two E-R mixed graphs as examples. A mixed graph of 12 points is established as in Figure 1 and a mixed graph of 34 points is established as in Figure 2. Then, we use the directed traversal algorithm (see Algorithm 2) to calculate $E_{\max }$ in the graphs, and we show the result in Table 1. 
Input: The adjacency matrix $A_{n \times n}$

Output: The Hermitian adjacency matrix $H_{n \times n}$

(1) Let $O$ be a null matrix

(2) Let $T=\{-1,1,-i, i\}$

(3) Let $O_{00}=1$.

(4) Randomly select an element of $T \backslash\{-1\}$ for $O_{11}$

(5) for each $i$ in $(2, n)$ do

(6) Randomly select an element of $T$ for $O_{i i}$

(7) for each $j$ in $(0, i)$ do

(8) if $O_{j j}=-O_{i i}$ and $A_{i j}=1$ then

(9) $\quad T \backslash\left\{O_{i i}\right\}$

(10) Randomly select an element of $T$ for $O_{i i}$

(11) end if

(12) end for

(13) end for

(14) $\mathrm{H}=O A O^{*}$

Algorithm 1: The positive walk algorithm.

Input: The mixed graph $G$

Output: Edge set $E_{\max }$

(1) Random select an edge $e_{0}$ of the mixed graph $G$

(2) Obtain the Hermitian resistance matrix $H$ of $G-e_{0}$

(3) Calculate the $K\left(G-e_{0}\right)$ and initialize $K_{\max }=K\left(G-e_{0}\right)$

(4) for each edge $e \in E(G)$ do

(5) remove $e$ from $G$

(6) calculate $K(G-e)$

(7) if $K(G-e)>K_{\max }$ then

(8) $K_{\max }=K(G-e)$

(9) $\quad E_{\max }=\{e\}$

(10) else

(11) if $K(G-e)=K_{\max }$ then

(12) $\quad E_{\max }=E_{\max } \cup\{e\}$

(13) end if

(14) end if

(15) end for

Algorithm 2: The directed transversal algorithm.

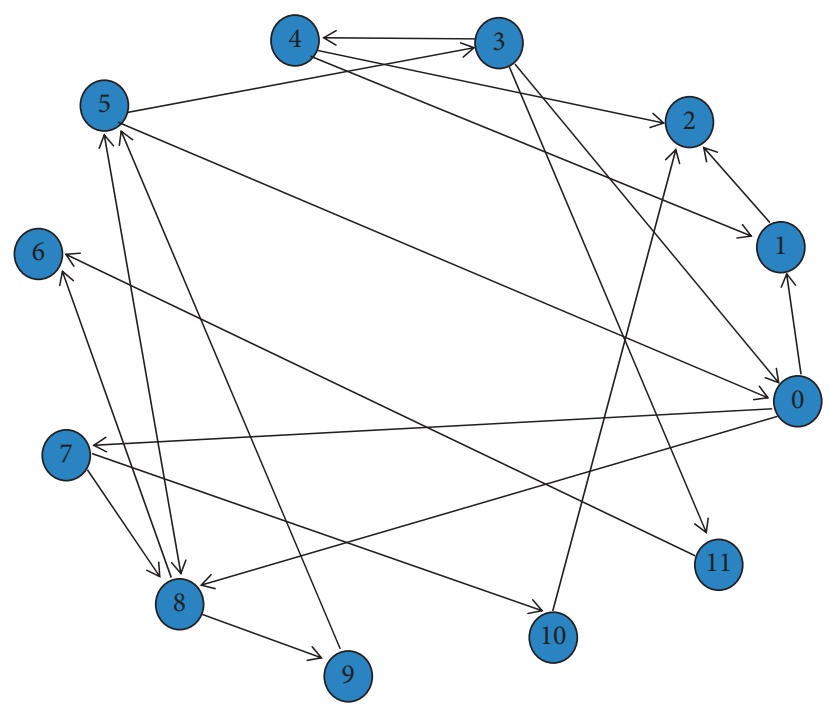

Figure 1: A mixed graph of 12 points. 


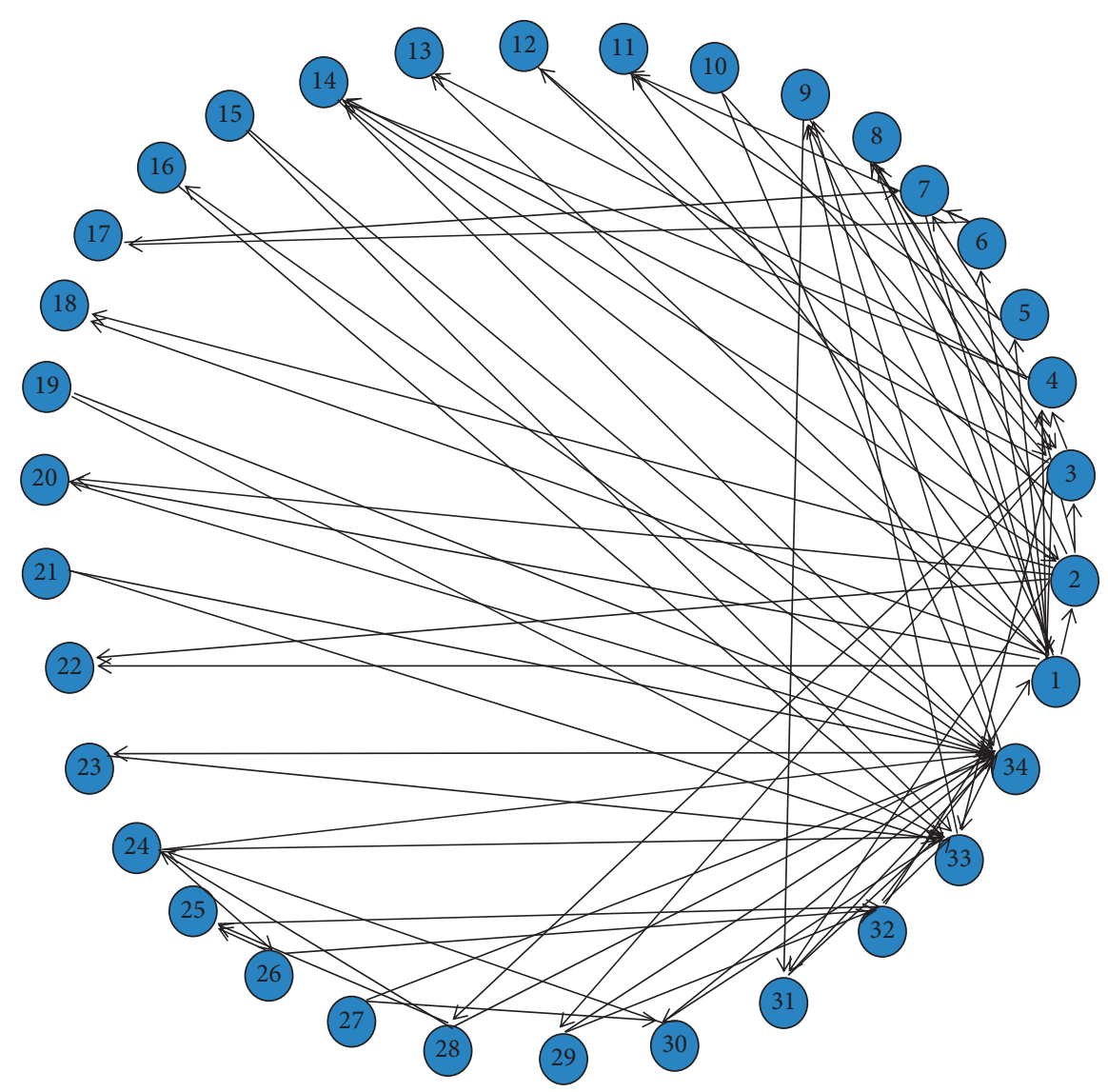

Figure 2: A mixed graph of 34 points.

TABle 1: Data statistics of the Hermitian Kirchhoff index after the edge is removed.

\begin{tabular}{lccc}
\hline Mixed graph & Removed edge & $K_{\max }$ & $\lambda_{\min }$ \\
\hline$G_{12}$ & $(5,8)$ & 139.2550 & 0.1381 \\
$G_{34}$ & $(17,7)$ & 470.9381 & 0.5091 \\
\hline
\end{tabular}

\section{Conclusion}

Many strategies of community detection in social networks have been developed, among them the spectral clustering based on the resistance matrix is widely used in community detection. In this paper, we focus on the Hermitian resistance matrix for positive mixed graphs and general mixed graphs. The Hermitian resistance matrix is a generalization for the resistance matrix in underlying graphs. In addition, we derive the formulae for the determinant, inverse of the Hermitian resistance matrix, and obtain the Hermitian Kirchhoff index. In order to show the practicability of the Hermitian matrix, we utilize the Hermitian Kirchhoff index to analyze the robustness of general mixed networks. Furthermore, we put forward the positive walk algorithm and the directed graph traversal algorithm. Our future work will apply the Hermitian matrices on algorithms for mining key nodes of directed networks and community detection.

\section{Data Availability}

The data used to support the study are included within the article.

\section{Conflicts of Interest}

The authors declare that they have no conflicts of interest.

\section{Acknowledgments}

This work was partly supported by National Natural Science Foundation of China (nos. 61977016 and 61572010), Natural Science Foundation of Fujian Province (nos. 2020J01164, 2017J01738, and JAT170118), Middle-aged and Young of the Education Department of Fujian Province of China (nos. JAT200958 and JAT191119), and Research Foundation of Concord University College Fujian Normal University (nos. KY20200204). 


\section{References}

[1] J. Jiang, B. An, Y. Jiang, and D. Lin, "Context-aware reliable crowdsourcing in social networks," IEEE Transactions on Systems, Man, and Cybernetics: Systems, vol. 50, no. 2, pp. 617-632, 2017.

[2] S. Rathore, P. K. Sharma, V. Loia, Y.-S. Jeong, and J. H. Park, "Social network security: issues, challenges, threats, and solutions," Information Sciences, vol. 421, pp. 43-69, 2017.

[3] Q. He, X. Wang, F. Mao et al., "CAOM: a community-based approach to tackle opinion maximization for social networks," Information Sciences, vol. 513, pp. 252-269, 2020.

[4] W. Lin, M. Li, S. Zhou, J. Liu, G. Chen, and Z. Gu, "Phase transitions in normalized cut of social networks," Physics Letters A, vol. 383, no. 25, pp. 3037-3042, 2019.

[5] X. Wang and G.-H. Yang, "Fault-Tolerant consensus tracking control for linear multiagent systems under switching directed network," IEEE Transactions on Cybernetics, vol. 50, no. 5, pp. 1921-1930, 2020.

[6] Y. Liu and G.-H. Yang, "Neural learning-based fixed-time consensus tracking control for nonlinear multiagent systems with directed communication networks," IEEE Transactions on Neural Networks and Learning Systems, vol. 32, no. 2, pp. 639-652, 2021.

[7] T. Wang, R. G. Qiu, M. Yu, and R. Zhang, "Directed disease networks to facilitate multiple-disease risk assessment modeling," Decision Support Systems, vol. 129, Article ID 113171, 2020.

[8] N. Alipourfard, B. Nettasinghe, A. Abeliuk, V. Krishnamurthy, and K. Lerman, "Friendship paradox biases perceptions in directed networks," Nature Communications, vol. 11, no. 1, pp. 1-9, Article ID 707, 2020.

[9] Z.-X. Cui, Q. Fan, and C. Jia, "Momentum methods for stochastic optimization over time-varying directed networks," Signal Processing, vol. 174, Article ID 107614, 2020.

[10] A. Tosyali, J. Kim, J. Choi, Y. Kang, and M. K. Jeong, "New node anomaly detection algorithm based on nonnegative matrix factorization for directed citation networks," Annals of Operations Research, vol. 288, no. 1, pp. 457-474, 2020.

[11] Z. Wu, Z. Di, and Y. Fan, "An asymmetric popularity-similarity optimization method for embedding directed networks into hyperbolic space," Complexity, vol. 2020, no. 5, 16 pages, Article ID 8372928, 2020.

[12] B. M. F. de Resende and L. F. Costa, "Characterization and comparison of large directed networks through the spectra of the magnetic Laplacian," Chaos, vol. 30, no. 7, Article ID 073141, 2020.

[13] D. J. Klein and M. Randić, "Resistance distance," Journal of Mathematical Chemistry, vol. 12, no. 1, pp. 81-95, 1993.

[14] W. Xiao and I. Gutman, "Resistance distance and Laplacian spectrum," Theoretical Chemistry Accounts: Theory, Computation, and Modeling (Theoretica Chimica Acta), vol. 110, no. 4, pp. 284-289, 2003.

[15] H. Chen and F. Zhang, "Resistance distance and the normalized Laplacian spectrum," Discrete Applied Mathematics, vol. 155 , no. 5, pp. 654-661, 2007.

[16] F. Atik, R. B. Bapat, and M. Rajesh Kannan, "Resistance matrices of graphs with matrix weights," Linear Algebra and Its Applications, vol. 571, pp. 41-57, 2019.

[17] W. Lin, M. Li, S. Zhou, J. Liu, G. Chen, and Q. Zhou, "Phase transition in spectral clustering based on resistance matrix," Physics A, vol. 566, Article ID 125598, 2021.

[18] M. S. Sardar, X.-F. Pan, and S.-A. Xu, "Computation of resistance distance and Kirchhoff index of the two classes of silicate networks," Applied Mathematics and Computation, vol. 381, pp. 323-336, Article ID 125283, 2020.

[19] S. Patterson, Y. Yi, and Z. Zhang, "A resistance-distancebased approach for optimal leader selection in noisy consensus networks," IEEE Transactions on Control of Network Systems, vol. 6, no. 1, pp. 191-201, 2019.

[20] K. Thulasiraman, M. Yadav, and K. Naik, "Network science meets circuit theory: resistance distance, Kirchhoff index, and foster's theorems with generalizations and unification," IEEE Transactions on Circuits and Systems I: Regular Papers, vol. 66, no. 3, pp. 1090-1103, 2019.

[21] T. Zhang and C. Bu, "Detecting community structure in complex networks via resistance distance," Physica A: Statistical Mechanics and Its Applications, vol. 526, Article ID 120782, 2019.

[22] J. Liu and X. Li, "Hermitian-adjacency matrices and hermitian energies of mixed graphs," Linear Algebra and Its Applications, vol. 466, pp. 182-207, 2015.

[23] K. Guo and B. Mohar, "Hermitian adjacency matrix of digraphs and mixed graphs," Journal of Graph Theory, vol. 489, pp. 324-340, 2015.

[24] J. Zhou, Z. Jiang, and S. Wang, "Laplacian least learning machine with dynamic updating for imbalanced classification," Applied Soft Computing, vol. 88, Article ID 106028, 2020.

[25] Y. Yang, L. Tu, T. Guo, and J. Chen, "Spectral properties of Supra-Laplacian for partially interdependent networks," Applied Mathematics and Computation, vol. 365, Article ID 124740, 2020.

[26] X. Ma, B. Zhang, C. Ma, and Z. Ma, "Co-regularized nonnegative matrix factorization for evolving community detection in dynamic networks," Information Sciences, vol. 528, pp. 265-279, 2020.

[27] J.-E. Byun and J. Song, "Efficient probabilistic multi-objective optimization of complex systems using matrix-based Bayesian network," Reliability Engineering and System Safety, vol. 200, Article ID 106899, 2020.

[28] B. Mohar, "A new kind of Hermitian matrices for digraphs," Linear Algebra and Its Applications, vol. 584, pp. 343-352, 2020.

[29] G. Yu, M. Dehmer, F. Emmert-Streib, and H. Jodlbauer, "Hermitian normalized Laplacian matrix for directed networks," Information Sciences, vol. 495, pp. 175-184, 2019.

[30] G. Yu and H. Qu, "Hermitian Laplacian matrix and positive of mixed graphs," Applied Mathematics and Computation, vol. 269, pp. 70-76, 2015.

[31] G. Yu, X. Liu, and H. Qu, "Singularity of Hermitian (quasi-) Laplacian matrix of mixed graphs," Applied Mathematics and Computation, vol. 293, pp. 287-292, 2017.

[32] B. Kang, K. Wu, Z.-W. Yan, J. Yang, and W.-Z. Zhao, "Exact correlators in the Gaussian Hermitian matrix model," Physics Letters B, vol. 798, Article ID 134986, 2019.

[33] M. Dehghan and A. Shirilord, "A generalized modified Hermitian and skew-Hermitian splitting (GMHSS) method for solving complex Sylvester matrix equation," Applied Mathematics and Computation, vol. 348, pp. 632-651, 2019.

[34] N. Truhar, Z. Tomljanović, and R.-C. Li, "Perturbation theory for Hermitian quadratic eigenvalue problem $\mathrm{C}$ damped and simultaneously diagonalizable systems," Applied Mathematics and Computation, vol. 371, Article ID 124921, 2020.

[35] S. Bermudo, J. E. NRandiápoles, and J. Rada, "Extremal trees for the Randić index with given domination number," Applied Mathematics and Computation, vol. 375, Article ID 125122, 2020. 
[36] C. T. Martínez-Martínez, J. A. Méndez-Bermúdez, J. M. Rodríguez et al., "Computational and analytical studies of the Randić index in Erdös-Rényi models," Applied Mathematics and Computation, vol. 377, Article ID 125137, 2020.

[37] C. Dalfó, "On the Randić index of graphs," Discrete Mathematics, vol. 342, no. 10, pp. 2792-2796, 2019.

[38] P. De Meo, F. Messina, D. Rosaci, G. M. L. Sarne, and A. V. Vasilakos, "Estimating graph robustness through the randic index," IEEE Transactions on Cybernetics, vol. 48, no. 11, pp. 3232-3242, 2018.

[39] Y. Peng, J. Li, and W. He, "Estimating robustness through Kirchhoff index in mesh graphs," IEEE Access, vol. 8, pp. 111822-111828, 2020. 